\title{
ZUR DEUTSCH-BRASILIANISCHEN MISCHSPRACHE
}

Reinhold Bossmann

Universidade do Paraná

Mit der Ankunft der ersten deutschen Einwanderer, im Jahre 1824, fasste die deutsche Sprache in Brasilien festen Fuss. Vor diesem Zeitpunkt kann das Erklingen der deutschen Sprache auf brasilianischem Boden nur als Einzelerscheinung bewertet werden. Die Sprachträger waren Forscher, Wissenschaftler, Kaufleute, Soldaten in fremdem Solde, Abenteurer und Strafverbannte. Von ihnen vernahm die einheimische Bevölkerung die ersten deutschen Laute. Doch mit ihnen verstummten auch sie, ohne einen sprachlichen Niederschlag zu hinterlassen. Erst die planmässigen Wellen deutscher Ansiedler, seit 1824, und Einwanderer als Einzelgänger verpflanzten die deutsche Sprache in die Wahlheimat.

Da die Einwanderer Angehörige der verschiedensten deutschen Stämme und Landschaften waren, wurden einige deutsche Dialekte durch sie nach Brasilien übertragen. Der Anteil der Schriftsprache bleibt dabei hinter den Mundarten zurück, wenigstens in der Anfangszeit der deutschen Kolonisation. Wo Kolonisten einer Mundart geschlossen siedelten, konnte sich diese noch verhältnismässig rein erhalten, besonders in der ersten Generation und, mit Einschränkungen, bis zur zweiten Generation. War das Siedlungsgebiet kein mundartlich einheitliches, die sprachliche Berührung und Vermischung mit anderen deutschen Dialekten gegeben, so setzte sich im allgemeinen die am meisten gesprochene Mundart durch. Für den Fortbestand einer Mundart in ihrer Rein- 
heit ist, neben anderen Faktoren, auch die Isolierung des betreffenden Sprachgebietes von der Umwelt entscheidend. Ist einmal für so ein Gebiet der Anschluss an das Verkehrsnetz vollzogen, beginnt die sprachliche Unterwanderung und damit das langsame Absterben der Mundart, im fremdsprachlichen Raum das der schwächer vertretenen Sprache. In diesem Fall entsteht eine Mischsprache als Zwischenstufe.

Nach Brasilien wurden durch die deutschen Kolonisten hauptsächlich folgende Mundarten im stärkeren oder schwächeren Grade übertragen: Pommerisch, Westfälisch als Untergruppen des Niederdeutschen; Fränkisch (Rhein-Moselfränkisch, Ripuarisch), Hessisch, Thüringisch, Obersächsisch, Ostmitteldeutsch als Untergruppen des Mitteldeutschen; Alemannisch, Schwäbisch, Bairisch, Oes terreichisch als Untergruppen des Oberdeutschen. Von diesen Mundarten ist heute fast keine mehr rein erhalten. Was noch existiert, sind lediglich kümmerliche Reste. Genaueres lässt sich darüber, mangels an Arbeiten, Teiluntersuchungen über die sprachliche Zusammensetzung in bestimmten Orten ${ }^{1}$ ) und Gegenden, Dialektaufnahmen usw., nicht sagen. Nach dem 2. Weltkriege wurden die aus dem Banat und der Batschka vertriebenen Donauschwaben in der Gegend von Guarapuava, im Staate Paraná, beheimatet. Seit 1718, ihrer Ansiedlung im Balkanraum, haben die Donauschwaben sich ihren Dialekt im grossen und ganzen, inmitten von andersvölkischen Stämmen, erhalten können. Es wird Aufgabe der Sprachwissenschaft sein, das weitere Schicksal dieser Mundart in Brasilien zu beobachten.

Wie gesagt, wurde die deutsche Schriftsprache nicht in so starkem Masse wie einige Dialekte nach Brasilien übertragen. Das gilt besonders für die Anfangszeit der deutschen Einwanderung. Die ersten Kolonisten, meistens Bauern, Handwerker, sprachen nur ihren Heimatdialekt. Geistige Berufe und der Mittelstand, deren Sprache das Neuhoch-

1) Für den Ort São Bonifácio, im Staate Santa Catarina, liegt eine Untersuchung vor: Egon Schaden, Aculturação linguistica numa comunidade rural, in: Sociologia, Vol. IV, Nr. 3, Săo Paulo 1942, S. 268-283. 
deutsche war, waren nur schwach vertreten ${ }^{2}$ ). Die Hochsprache gewann dann stärkeren Einfluss in den verschiedensten Phasen der Immigration, als deutsche Schulen eingerichtet, deutsche Lehrer bestellt wurden, als deutsche Zeitungen, Zeitschriften ") und Schulbücher für die Kolonisten erschienen. Die segensreiche Tätigkeit der Kirchen, auch für die Verbreitung der Schriftsprache und den Fortbestand der Muttersprache unter den Kolonisten, ist dabei nicht hoch genug einzuschätzen. Die Stellung der deutschen Hochsprache wurde durch jeden Einwanderer aus den gebildeten Kreisen gestärkt. Trotzdem behaupteten die Mundarten immer ihre dominierende Position. Auch der wirtschaftliche und kulturelle Aufschwung in Städten und Dörfern liess sie nicht verstummen.

So wies die sprachliche Situation der deutschen Einwanderung nie ein einheitliches Bild auf. Die Schriftsprache hatte keine vorherrschende Stellung, die Mundarten waren zu vielfältig. Daher fehlte für die Bildung einer deutschen Verkehrssprache, im Sinne einer Umgangssprache von Kolonie zu Kolonie, von Stadt zu Land, schon jede Voraussetzung. Diese Tatsachen waren mitentscheidend für das verhältnismässig schnelle Eindringen des Portugiesischen in die hier gesprochene deutsche Sprache. Begünstigt und gefördert wurde die sprachliche Unterwanderung, einmal durch die Notwendigkeit für die Kolonisten, sich den neuen Verhältnissen auch sprachlich anzupassen, zum andern durch den vermutlich geringen Wortschatz in der Muttersprache. Wie jedes Werden, jede Geburt schon die Keime des Todes in sich trägt, so war die Geburtsstunde der deutschen Sprache im fremdsprachlichen brasilianischen Raum schon der Beginn für ihr langsames, wenn auch zähes Abklingen. Es ist schliesslich, wie jede Assimilation, ein ganz natürlicher Vor-

2) Ein genaues Bild von der deutschen Einwanderung nach Brasilien, mit allen damit zusammenhängenden Fragen, von den sozialen Verhältnissen der Einwanderer, von ihrer Schichtung nach Berufen, gibt Emilio Willems in seinem ausgezeichneten Werk "A aculturação dos alemães no Brasil", São Paulo 1946, 609 S.

3) Die meisten allerdings waren auf die bäurische Bevölkerung zugeschnitten und erreichten kaum ein durchschnittliches Niveau. 
gang, dass die zahlenmässig schwächer vertretene Sprache von der stärkeren nach und nach durchdrungen und aufgesaugt wird. Das Zwischenprodukt dieses Prozesses ist eine Neben- oder Mischsprache, ein Gemisch von zwei oder mehreren Sprachen, die in einem Raum in ständiger Berührung sind, sich überschneiden und ineinandergreifen. Der Grad ihrer Mischung steigt mit dem Fehlen an Bildung, an Intelligenz ihrer Träger, an Sprachdisziplin, mit der Gedankenlosigkeit beim Sprechen, mit dem Mangel für jegliches Formund Schönheitsgefühl einer Sprache; er fällt mit der Bildung, Geistesschärfe, Selbstzucht beim Sprechen und dem Willen nach einem guten und einwandfreien Ausdruck. Das Zusammenwirken all jener negativen Kräfte schafft die Voraussetzung für die sprachliche Vermischung, die Einengung des Wortschatzes in der Muttersprache und so das notwendige Uebel, immer mehr fremde Wörter aufzunehmen.

Der geringe Wortschatz in der Muttersprache der ersten Einwanderer genügte nicht, um mit der neuen Umgebung in sprachlicher Hinsicht fertig zu werden. Die für sie fremde Flora, Fauna, vorher nicht gekannte Werkzeuge, Dinge des täglichen Gebrauchs, Lebensmittel, Speisen, kurz, die totale Umstellung der gewohnten Lebens- und Arbeitsweise, die Anpassung an Land und Leute, an die Gesellschaft und den Staat, forderten gebieterisch nach einer Benennung ${ }^{4}$ ). Es galt nun, entweder mit den eigenen sprachlichen Mitteln auszukommen, oder sprachliche Anleihen aufzunehmen. Beide Wege wurden beschritten; auch die sprachliche Phantasie sorgte für den Kern der Sache treffende Neuschöpfungen in der Muttersprache. Einige Entlehnungen wurden übersetzt oder abgeändert, d. h. der deutschen Sprache angepasst, andere direkt unverändert übernommen. Dazu einige Beispiele nach Willems ${ }^{5}$ : eine Bambusart, als Fackel gebraucht, be-

4) Willems, a.a.O. S. 277, gibt drei Gründe für den Wandel der Sprache in teutobrasilianischen Orten an: 1) Die Lücke im Wortschatz auszufüllen, bedingt durch die verschiedenartige Struktur der alten und der neuen Heimat; 2) der kulturelle Unterschied und die Vielfalt der Mundarten unter den Kolonisten; 3) die Symbiose mit Gruppen von verschiedenartiger Kultur.

5) a.a.O. S. 278 f. 
kam die Bezeichnung Lichtrohr (cana para luz) in São Leopoldo; der Ortsname Estrela $>$ Strehle im Munde der Kolonisten; estância $>$ Stanz; foice $>$ Fuchs (feuss', fouss); roçar $>$ rossen.

So sind schon vom Beginn der deutschen Kolonisation zahlreiche portugiesische und indianische Wörter in die Sprache der Kolonisten eingegangen. Es wäre falsch, den Grund dafür nur in der Notwendigkeit suchen zu wollen, die vorhandene Lücke im Vokabular der Muttersprache auszufüllen. Häufig war es der Wunsch, das eigene Interesse, soviel als nur möglich von der fremden Sprache sich anzueignen, die Sprachklippe zu überwinden, um am Leben in der neuen Heimat regen Anteil zu nehmen. Dieses Bemühen erscheint um so verständlicher, wenn der Kontakt mit der einheimischen Bevölkerung gegeben $u$. die Kenntnis der Landessprache auf allen $Z$ weigen des öffentlichen Lebens nur von Vorteil, ja unumgänglich nötig war. Dass allerdings eine solche Sprachbereicherung sich zum Nachteil der Muttersprache auswirkte, ist freilich eine traurige, aber immerhin naturgegebene Feststellung im Ablauf des sprachlichen Assimilierungsprozesses. Er wird augenscheinlicher von Generation zu Generation unter den Brasilianern deutscher Abkunft.

Emilio Willems ${ }^{6}$ ) bietet in seinem Werk eine Liste der portugiesischen Wörter im Sprachgebrauch der Teutobrasilianer. Die Liste ist alphabetisch angelegt. Danach benutzen die Deutschstämmigen in sprachlich isolierten Gegenden, wo der Aufbau von Sprachgemeinschaften möglich war, 693 Termini. Davon sind nur 37 Verben, 6 Adjetive und 8 Interjektionen 7). Von den Verben sagt Willems wörtlich: "Mit einer einzigen Ausnahme haben alle Verben die germanischen Endungen erhalten (-ieren, -en) 8). Dazu ist zu bemerken, dass die Endung -ieren nicht germanischen, sondern romanischen Ursprungs ist: -ieren< frz. -ier+dt. -en; (frz. -ier $<$ lat. -iare, -igare). Mit der französischen Ritterdichtung dringt sie ins Mittelhochdeutsche ein. Vielleicht hat Willems

6) a.a.O. S. $281-97$.

7) Willems, a.a.O. S. 298.

8) Willems, a.8.O. S. 298. 
unter ,germanischer" Endung auch nur ihr blosses, harmloses Aeussere gemeint. Im Vokabular von Willems befinden sich viele Wörter, die indianischen Ursprungs, also keine portugiesischen Wörter sind. Die meisten gehören dem Tupi und Guarani an. Als Beispiel seien nur einige angeführt: aipim, araçá, arapuca, beijú, goiaba, guri, capim, capoeira, capão u. a. Freilich haben sie die Kolonisten durch portugiesische Vermittlung, nicht direkt von den Indianern, angenommen. Trotzdem ist der Titel des Wörterverzeichnisses "Lista de palavras portuguesas incorporadas no linguajar teuto-brasileiro" als nicht besonders glücklich gewählt $\mathrm{zu}$ betrachten, da diese Wörter nicht zum reinen portug. Sprachschatz gehören, während sie der Brasilianer als seine eigenen ansieht. Dasselbe gilt für das Vokabularium von C. H. Oberacker ${ }^{9}$ ) das bei geringerem Umfange, z. T. dieselben Wörter wie das von Willems enthält. Oberacker ${ }^{10}$ ) macht aber im Text eine Erweiterung. Leider sagt Willems nichts, Oberacker selten und nur allgemein, über die Verbreitung, Häufigkeit und über den Grad ihrer Verteilung in der Bevölkerung. Die Angaben über die Literatur der Verbreitung hätte man bei Willems erwarten können, zumal es einführend $z u$ seinem Vokabularium heisst: "Foram colhidos até agora 693 têrmos que os teuto-brasileiros introduziram no idioma falado em áreas isoladas onde a constituição de comunidades lingüísticas era possível“. ${ }^{11}$ )

Willems hat in sein Verzeichnis einige Wörter aufgenommen, die wahrscheinlich nicht über das Portugiesische in den Sprachschatz der Kolonisten gelangten, sondern die schon zu ihrem Sprachgut in der Heimat gehörten. Solche Wörter 12) sind: adyés, aksizen, aranxiren, balkon, biskút, despaxiren, distrikt, garafe, karáf, inkomodiren, inspektor, kamarát, kamer, kapitál, kartonaxe, katúx, koloní, konver-

9) Vocabulário de palavras portuguesas que os descendentes de colonos alemães acolheram na língua vulgar, in: Sociologia, Vol. 1, N. 3, São Paulo 1939, S. 96-104.

10) a.a.O. 5. 97: Abaixo publicaremos um pequeno vocabulário de palavras portuguesas ou indígenas...

11) Willems, a.a.O. S. 280.

12) Die Art ihrer schriftlichen Wiedergabe erfolgt hier nach Willems. 
siren, laso, latrine, liberaler, limonade, maxor, mulát, pasaxe, prefektúr, republikaner, salon.

$\mathrm{Zu}$ adyés: in die deutschen Mundarten ist es als adis, adjes nach dem Vorbild von bona dies im 18. Jh. eingedrungen, wurde im Südwesten Deutschlands zu átje, in Berlin zu tsch ̈̈, in Brasilien als tchau (wahrscheinlich vom Italienischen her) gebraucht; adieu < frz. dieu, dieses < lat. deus.

$\mathrm{Zu}$ aksizen: diese Form weist deutlich hin auf Akzise, Plur. Akzisen, im späten Mittelalter aus dem Lateinischen ins Deutsche eingegangen.

Zu balkon: im Sinne von Ladentisch, Verkaufstisch im Geschäftslokal, ist die portugiesische Vermittlung gegeben, als Erker, Fenster und Raum im Theater unwahrscheinlich, da das Wort im 17. Jh. ins Deutsche rückwandert.

Die Wörter Biskuit, Distrikt, Karaffe, Inspektor, Kamerad, Kammer, Kartonage, Kartusche, Kolonie, Lasso, Latrine, Liberaler, Liga, Limonade, Major, Mulatte, Pasșage, Republikaner, Salon, Zeremonie sind im Deutschen lange vor dem Beginn der deutschen Auswanderung nach Brasilien, einige seit Jahrhunderten, belegt. Fast alle sind Termini der Umgangssprache und so allgemeinen Charakters, dass sie vielleicht zum Wortschatze jedes deutschen Auswanderers gehört haben. Das Wort kapital ${ }^{1: 3}$, eriäutert Willems nur durch capital, ohne zu sagen, was damit gemeint sei. Angelegte Gelder oder Hauptstadt? Kapital für Vermögenswerte kennt das Deutsche schon seit dem $17 \mathrm{Jh}$., für Hauptstadt kann es als Worteigentümlichkeit ${ }^{1+}$ ) der portugiesischen Sprache zur deutschen gelten. Die gemachten Einschränkungen zu einigen Wörtern in der Liste von Willems gelten auch für das Vokabularium von Oberacker: bei Jasmin ${ }^{15}$ ) und Chef kommt kaum eine Vermittlerrolle der portugiesischen Spra-

13) Willems, a.a.O. S. 287.

14) In diesem Zusammenhange sei verwiesen auf den Entwurf zu einem Handbuch „Worteigentümlichkeiten der brasiliarischen Sprache“ von Erich Arnold von Buggenhagen, erschienen als Veröffentlichung des Instituto Hans Staden, São Paulo 1951, 164 S. Mit dem Erscheinen des Wörterbuches der Worteigentümlichkeiten, unter der Redaktion von E.A. v. Buggenhagen, ist wohl im Laufe des Jahres $1954 \mathrm{zu}$ rechnen.

15) Oberacker, a.a.O. S. 97-98. 
che in Frage, höchstens in der Aussprache des ,j", beim zweiten in der Hinzufügung des ,e“ als Endung (chefe).

In den letzten Jahren gewann das Portugiesische als Nationalsprache immer mehr Boden in der Umgangssprache der Teutobrasilianer. Die Menge der portugiesischen Wörter, der täglichen Redewendungen, in hier gedruckten Büchern, Schriften und in der Presse veranschaulichen, wie weit der sprachliche Assimilierungsprozess schon fortgeschritten ist $\left.{ }^{16}\right)$. Die folgende Sammlung fusst nicht auf einer bestimmten Sprachlage; sie berücksichtigt nur die Umgangssprache, die Sprache des Alltags; ihre horizontale Reichweite ist nicht abgesteckt, d. h. sie führt nur allgemeines Sprachgut, keine Regionalismen auf. Nach ihrer vertikalen Reichweite hin basiert sie auf der Allklassigkeit, nicht auf einer bestimmten Gruppen- oder Standessprache. Die zu diesem Thema bereits vorliegende Literatur ist noch nicht so umfangreich, aber immerhin einschlägig; sie ist, soweit sie für den Verfasser dieses Artikels erreichbar war, am Ende aufgeführt. Bereits zu dieser Frage veröffentlichtes Material wurde hier nicht aufgenommen. Für den Leser ausserhalb Brasiliens ist dem Portugiesischen die deutsche Uebersetzung in Klammern beigegeben. Eine Phonetisierung ist hier aus drucktechnischen Gründen nicht möglich.

\section{Benennungen aus dem Bereich der Technik:}

liquidificador (Starmix, Küchenmaschine), geladeira (Eisschrank), refrigerador (Kühlschrank), fogão (Herd, Küchenofen), fogão econômico (Sparherd), estufa (elektrischer Heizofen, Heizkörper), válvula (Radioröhre, Ventil), parafuso, Parafuse (Schraube), disco (Schallplatte), discoteca (Schallplattensammlung), toca-disco (Plattenspieler), disco voador (fliegende Untertasse), gaita (Schifferklavier), Fôrça e Luz (wörtl. Kraft und Licht, hier: Elektrizitätsgesellschaft), lâmpada fluorescente (Neonlicht), fita (Farbband der Schreibmaschine), fita isolante (Isolierband), prego (Nagel), aço (Stahl),

16) Auf die Grinde, die dazu flhrten, năher einzugehen, wlirde den Rahmen dieser Arbelt oprengen. 
casa do aço (Geschäft für Stahlwaren), metalúrgica (Metallwarenfabrik), água e esgôto (Wasser und Kanalisation), cortume (Gerberei), chuveiro (Brause, Dusche), assistência técnica (technische Hilfe), tipografia (Druckerei), paginação (Umbruch beim Druck), construtora (Baufirma), alto-falante (Lautsprecher), polegadas (Zoll), ôlho mágico (magisches Auge), pedreira (Steinbruch), poste (Mast, Laternenpfahl), fogareiro de querosene (Petroleumkocher), usina (Kraftwerk), cafeteira de vidro (Kaffeemaschine aus Glas), resistente (widerstandsfähig), máquina de escrever portátil (Reiseschreibmaschine).

\section{Aus Handel, Gewerbe und Beruf:}

registrado (eingeschrieben), caneta (Federhalter), negócio (Geschäft), presente (Geschenk), companhia (Gesellschaft), avulso (einzeln, Verkauf nach Stück), relatório (Bericht), pedido (Bestellung), freguês (Kunde), orçamento (Kostenanschlag), empréstimo (Darlehen), acréscimo (Erhöhung, Aufschlag), carga (Fracht), reembôlso (Nachnahme), artigo (Artikel), sociedade anônima (Aktiengesellschaft), sociedade limitada (Gesellschaft m. b. H.), recibo (Empfangsschein, Quittung), assinatura (Unterschrift, Abonnement), cópia (Durchschlag), inteiro (ganzes Lotterielos), fração (Teillos, $1 / 10$ Los), à vista (auf Barzahlung), auf prestações (Ratenzahlung), contabilidade (Buchhaltung), contador (Buchhalter), coletor (Einkassierer, Einnehmer), corretor (Makler), frios (Aufschnitt), Frios-Geschäft, fiador (Bürge, Gewährsmann), contrato (Vertrag), depósito (Hinterlegung, Pfand, Geldeinzahlung), depósito (Magazin, Vorratsraum), diretor, gerente (Geschäftsführer), dúzia (Dutzend), lucro (Reingewinn), amostra (Muster, Probe), jôgo (Satz, Garnitur, Spiel), poltrona (Sessel), cofre (Geldschrank), tropical (leichter Anzug für die Tropen), pagamento (Zahlung), prazo de pagamento (Zahlungsfrist), seguro (Versicherung), leilão (Versteigerung), aluguel (Miete), loção (Haarwasser), algodão (Watte), nata batida (Schlagsahne), marmita (Menage, Einsatzschlüssel), sócio (Teilhaber), proprietário (Inhaber, 
Eigentümer), vitrina (Schaufenster), imposto (Steuer), vale (Kassenanweisung), vale postal (Postanweisung), caixa postal (Postschliessfach), casaco (Jacke), tubarão (Haifisch, im Sinne für Schwarzhändler), remessa (Sendung), requerimento (Gesuch), petição (Gesuch), confiança (Vertrauen), dicionário (Wörterbuch), editor (Verleger), editora (Verlag), prova (Korrekturbogen), amador (Amateur), profissional (Berufs-), maternidade (Entbindungsheim), consultório (Sprechzimmer des Arztes), instituto de belêza (Damensalon), lavanderia (Wäscherei, Waschanstalt), caça (Jagd), representante (Vertreter), cirurgião (Chirug), dentista (Zahnarzt), parteira (Hebamme), enfermeiro, enfermeira (Krankenpfleger, Krankenschwester), lavadeira (Waschfrau), empregada (Dienstmädchen), guarda (Wächter), caçador (Jaeger), barbeiro (Haarschneider, auch für einen Stümper, z. B. einen schlechten Kraftfahrer, barbeiro!), ladrão (Dieb), ' bombeiro (Feuerwehrmann), benzedeira (Gesundbeterin, Quacksalberin).

\section{Aus dem Verkehrswesen:}

farol (Leuchtturm), fila (Reihe, Schlange) in der Fila stehen, empresa (Verkehrs-, Transportunternehmen), expresso (Express, schnelles Fahrzeug), rápido (schnelles Fahrzeug), horário (Fahrplan, Arbeitszeit, Stundenplan), Praça-Auto (Taxi), lotação (kleines Verkehrszeug für eine geringe Anzahl von Fahrgästen), macadame 17) (Wegversteinung), makadamisieren, movimento (Verkehr, Betrieb), parada (Haltestelle), fim da linha (Endstation), choque (Zusammenstoss), barulho, (Lärm, Krach), aeroporto (Flughafen), aerodromo (Flugplatz), avião (Flugzeug), faixa 18) (Streifen, Markierung zum Strassenübergang für den Fussgänger), condução (Fahrgelegenheit), cerração (Nebel), travessa (Querstrasse), quadra (Häuserblock, Ecke).

17) nach dem schottischen Wegebau-Inspektor Mac Adam.

18) Der Volksmund benennt auch so eine Strasse in Porto Alegre, im oberen Stadtteil von Petrópolis. 


\section{Aus Haus und Hof:}

edifício (Gebäude), andar (Stockwerk), casa de aluguel (Mietshaus), apartamento (Appartement, Wohnung), sala de jantar (Esszimmer), copa (Speisezimmer), dispensa (Speisekammer), dormitório (Schlafzimmer), privada (Toilette, Lokus), porão (Keller), armário (Schrank), cristaleira (Glasschrank), prateleira (Regal), guarda-comida (Speiseschrank), quintal (kleiner, ummauerter Garten), lenha (Brennholz), mosquiteiro (Moskitonetz), almofada (Kopfkissen), corda (Schnur, Strick, Seil), für ein casal (Ehepaar), für einen solteiro (Junggesellen), lixo (Kehricht, Müll).

\section{Aus Kirche und Schule:}

arcebispo (Erzbischof), bispo (Bischof), batina (Soutane, langer Rock des Geistlichen), missa (Messe), missa campal (Feldmesse), promessa (Gelübte), confissão (Beichte), colégio (Gymnasium, höhere Schule), professor (Lehrer), lente (Universitätsprofessor), catedrático (Inhaber eines Lehrstuhls), grupo escolar (Volksschule), primário (Volksschule), secundário (höhere Schule), superior (Universitätsausbildung), aula (Unterricht, Klasse), conferência (Vortrag), turma (Abteilung, Schulklasse), reunião (Zusammenkunft, Sitzung, Konferenz), boletim (Zeugnis), sabatina (Klassenarbeit), prova (Klassenarbeit), trabalho prático (Uebungsarbeit), aulas particulares (Privatstunden), mata-borrão (Löschblatt), mapa (Landkarte), tradução (Uebersetzung), versão (Uebersetzung), bolsa (Stipendium I, feriado (Feiertag, schulfrei), pasta (Büchertasche, Aktentasche).

\section{Aus verschiedenen Bereichen:}

vizinho (Nachbar), namorado, namorada (Liebster, Schatz, Liebchen), moreno, morena (dunkelbraun, dunkler Typ), vereador (Stadtverordneter, Stadtrat), bobo (Dummkopf), burro (Esel), príncipe (Fürst, Prinz), carteira de identidade (Personalausweis), mala (Koffer), aniversário (Geburtstag), baile 
(Tanz, Ball, Vergnügen), regístro (Eintragung, Waffenschein), portátil (Erlaubnisschein zum Tragen einer Waffe), conto do vigário (Schwindelgeschichte, Schwindel, Betrug), mentira (Lüge), arrombamento (Einbruch), corrida (Wettrennen), interior (im Innern des Landes, auf dem Lande), centenário (Jahrhundertfeier), pronto (fertig, bereit), sotaque (Akzent, Klangfärbung in der Aussprache).

\section{Interjektionen und Exklamationen:}

nāo há dúvida (zweifellos), graças a Deus (Gott sei Dank), parabens (Glückwünsche), engraçado (komisch, unerhört), barbaridade (Gemeinheit), porcaria (Schweinerei), não vale a pena (nicht der Mühe wert), é, sim (ja, jawohl), pois é, (ja, jawohl, freilich), pois não (warum nicht? ja doch, gewiss), felicidade ((viel Glück); isso (ja, gewiss), é duro (es ist schwer, hart).

\section{Portugismen in Redewendungen:}

Heute hat's keine Aula (hoje não há aula).-Heute hat's keine Kraft (für Strom; wörtl. Uebersetzung von fôrça). - Ich bin mit Eile (estou com pressa). Sie macht die erste Kommunion (faz a primeira comunhão). Er wird fünfzehn Jahre machen (vai fazer quinze anos). - Ich mache den Kaffee kochen (faço ferver). - Ich will mit ihm keine Freundschaft machen (fazer amizade). - Sie hat sich geheiratet (casou-se). - Im Merkado hat es viele Bankas. (Auf dem Markt gibt es viele Stände).

Die Durchsicht des Anzeigenteils in einer grossen deutschsprachigen Tageszeitung (,Deutsche Nachrichten“) erbrachte folgende Ausbeute:

Metallfüsse und sonstige Metallteile für padronisierte Möbel zu verkaufen. - Lassen Sie Ihre Joias bei dem Fachmann alt auf neu umarbeiten... - Terrenos zu verkaufen. Corretores am Ort... - Garage zu vermieten. Geeignet für Oficina oder Depósito... - Konserven, Frios, Hühner... Reparaturen von Radios, Vitrolas und Televisão... - Empregada für alle Hausarbeiten... - Junges Mädchen sucht Stellung als Arrumadeira... - Grosser Spezial-Ausverkauf. 
Profitieren Sie unsere Balkon-Offerten... - Gesucht wird ein Porteirc, mit guten Referenzen, vorzustellen im Frigorifico .. - Lojas, Armazens zu vermieten mit gutem Depot, W.C., Tank, geeignet für Mercearia, Kurzwaren-, Eisenwaren-, Fleischwaren-Geschäft, Apotheke oder anderen Geschäftszweigen. Haben Caminhão-Einfahrt... - Copeira gesucht für gepflegten Haushalt... - 50 Contos werden gesucht... Loten mit Front zur schönsten Represa von... - Grundstücke auf Teilzahlung... Gegenüber der Gasolinpumpe der Endstation des Omnibus Aeroporto. Angeschlossen dem Sindicato dos Corretores de Imóveis... - Vermiete erstklassig gebautes Haus mit Entrada, gr. Varanda, Salas... - Ebenerdiges Wohnhaus mit Bad, Brause-Box, Copa, Dispensa... zu vermieten... - Sehr guter Porão und komfortable Wohnung zu vermieten. Der Porão hat Tür und gute Fenster nach der Strasse... - Vermiete freistehendes Haus, enthält Besuchzimmer mit Kamin, gr. Esszimmer, 2 Schlafzimmer, CopaKüche, Bad, Despence u. Garage... - Eck-Sobrado zu vermieten, mit Kontrakt und Fiador. Nähe Aeroporto... Perfekter Korrespondent offeriert sich für portug. und engl. Korrespondenz... - Ausgezeichnete Grundstücke mit herrlicher Panorama-Aussicht, gelegen in dem fortschrittlichsten Stadtteil, mit prachtvollen Residenzen... - $\mathrm{Zu}$ verkaufen grosse Palacete mit Piscina... - Haus zu verkaufen, zwischen Omnibus- und Bondlinie, mit allem Komfort, Sala... - $\mathrm{Zu}$ vermieten ausgezeichnete Villa, freistehend, enthält: Living mit Lareira, Ess-Saal, vier Schlafzimmer, Copa-Küche u.s.w. Kontrakt und Fiador... - Lotes an der Praia, neue Loteamentos $_{\text {}}$ die schönsten, die Sie wohl sahen! Fahrt in Luxus-Omnibussen und Mittagessen offeriert der Besitzer. Bitte die Plätze bis Freitag zu reservieren...

Auf einem Bericht mit dem Titel "Milhofest in Santa Rosa“, veröffentlicht in der Zeitung „Deutsche Nachrichten“, São Paulo:

Jede Stadt feiert das Datum ihrer Gründung. Jede Stadt ist stolz auf die Höhepunkte ihrer Geschichte. Santa Rosa erlebte am 25. Juli sein Milhofest, vom Präsidenten der Re- 
publik offizialisiert, mit Staatsmitteln subventioniert. - Alles strebte in die Neustadt, wo die Ausmasse des Quartels festgelegt wurden, die einer Praça, ein imposantes Gebäude, in dem die Präfektur ihren zukünftigen Sitz haben würde. „Sind das wirklich Pflüge?" . . diese discus? „Sim, Senhor, das sind die modernsten ihrer Art." „.. und ist das wahr, o Sr., dass diese Pflüge zehn Quart am Tage umreissen?“ „Natürlich." Ein zufriedenes Aufleuchten ging über sein schwarzes Gesicht: „Ah, nun bin ich glücklich. Jetzt kommt noch mal für uns die goldene Sonne Zeit. Wisst Ihr, Senhor, ich hab' sieben Kinder zu ernähren und verdiene kaum 25 Cruzeiros am Tag. Und der Reis kostet 9 Cruzeiros. Ah, graças a Deus, jetzt wird es anders. Die Discus ... são da Fabrica alemã, não é?" "Não, vizinho, das sind echte brasilianische Discus." - „Vizinho," sage ich und bemühe mich um eine würdige Haltung: „Sie kennen doch das Wort Deus é brasileiro; seht, Mann, wenn Ihr den Glauben nicht verliert, dann wird der Reis schon billiger werden..." Tief Atem holend, murmelte er noch einmal wie zu sich selbst: „Agora sim, jetzt wird der Reis billiger und die schwarzen Bohnen und ein bisschen Xarque wird's dann auch mal wieder geben..."

Aus: „Die Geschichte eines Sturzes“ von Monteiro Lobato, aus der Skizzensammlung "Cidades Mortas", übersetzt von Dr. R. Löw 19):

Nur der Tod wirft von Zeit zu Zeit in die leere Büchse des Erlebens den verwitterten Vintem eines armen an Altersschwäche gestorbenen Fischers oder den Tostão einer Person von Stand, eines Collectors de Rendas, Fiscals oder Postagenten. - Das Getuschel der bösen Zungen, sei es im Familienzirkel, sei es in der Vende des José Inchado (Stelldichein der kleinen Leute) oder in der Botica zum goldenen Hai (Treffpunkt der höheren Gesellschaft), musste damit vorlieb nehmen... - Nun wird der Promotor die schmetternde Arie der Anklage singen; der Zézéca Estéves, der Solicitador, wird die "Verrückte von Albano" zum Vortrag bringen als

19) veröffentlicht im Kalender der „Serra-Post“ für das Jahr 1926, s. $46-52$. 
Verteidigung. - Erinnern Sie sich, Compadre, jener Jury,... - Der Pedro Intanha hatte einen Wortwechsel mit dem Major Vaz, verlor den Kopf und nannte ihn "estupor" (Dummschädel). - Der Uebeltäter zeichnete in der Luft einen kunstvollen verschlungenen Schnörkel, der des Vereadors Gleichgewicht erschütterte... - Der Escrivão flüsterte mir ins Ohr, dass es immer so war. - Ich las: „Herr Dr. Juiz, tun Sie uns entschuldigen, aber verurteilen den Kerl zur höchsten Strafe (no gráo maximo)...". Die Session ist aufgehoben! Der Cabo und die Soldaten begaben sich auf die Suche.

Aus dem „Riograndenser Musterreiter" ${ }^{\text {20 }}$ ):

Trotzdem die Kommission sogleich ihre Arbeit begann, konnte das Buch nicht bis Weihnachten 1911 fertiggestellt werden, da die allseitige Mitarbeit der Mitglieder com paciencia betrieben $u$. somit die Herausgabe auf die lange Bank geschoben wurde ${ }^{21}$ ). - Später etablierte er mit N. Lammers ein Fazendas-Geschäft, das er aber, weil unrentabel, rasch liquidierte ${ }^{22}$ ). - Auch ein Vereinsabzeichen: eine silberne Plakette, die Initialen M. C. tragend, mit blauweisser Schleife versehen, wurde angeschafft (das Protokoll nennt sie „,Schappe“) ${ }^{23}$ ).

Für die Damen gibt es auch liqueur oder Wasser mit Himbeersaft und Doce, das heisst süsses Backwerk ${ }^{24}$ ). - Man gibt für die ganze Nacht von einem Patação, das heisst 2 Milreis (1 Milreis = 1 Mark 25 Pf.) aufwärts ${ }^{25}$ ) — . . von wo er den Kundenkreis in unserer Fronteira auf ausgedehnten Reisen $z u$ besuchen hatte ${ }^{26}$ ). $-\ldots$ in einem verfallenen Sobrado... 27). - Eben steckt der Neger sich einen bleistiftlangen cigarro de palha (Maisstrohzigarette) an 28,

Den Brasilianismen sind im Musterreiterbuch meistens die

20) Herausgegeben vom "Musterreiter-Club" in Porto Alegre, Staat Rio Grande do Sul, Brasilien, Porto Alegre, 1913, 192 S.

21) aus der Vorrede zum Musterreiter.

22) Musterreiter, S. IX.

23) Musterreiter, S. 5.

24) Musterreiter, S. 13.

25) Musterreiter, S. 19.

26) Musterreiter, S. 37.

27) Musterreiter, S. 79.

28) Musterreiter, S. 95. 
entsprechenden Exklärungen beigegeben, wahrscheinlich zum besseren Verständnis für den deutschen Leser ausserhalb von Brasilien:

peão (Knecht), trôco (Wechselgeld), seccos und molhados (Trockenes und Nasses), potreiro (grosser mit Draht umzäumter Weideplatz), acampamento (Lager), partidas (Streifkorps), trabucos (Sattelpistolen), pampeiro (Sturm), tropeiro (Maultiertreiber), bombachas (Pluderhosen), Milhobrot (Maisbrot), cachaça (Zuckerrohrschnaps), bolaxas (Schiffszwieback), capão (Waldinsel auf dem Camp), saráu Tanz, Picapaos 29) (Regierungstruppen), Maragatos (Revolutionäre), caboclo (Haibindianer), vendinha (kleines Kaufhaus).

An Tautologismen verzeichnet der Musterreiter:

Pois é - natürlich, certamente - gewiss, é, sim, senhor - jawohl, pois não — warum nicht? sirva-se senhor bedienen Sie sich!

Gesprächsproben aus dem Musterreiter-Buch:

„te logo, Wilm, pass bem! - Ja, wenn du meinst -_.. „Ja, ja, das mit der Sprache ist die pure Verdade,“ sagte ein São Leopoldenser Kaufmann, „besonders Zahlen versteht der Lorenz schlecht; wenn ich 5 Peças Schita bestelle, sendet er infallivelmente por minimo 10." „Sing Senjor, Herr Farbenecht, das ist nun einmal so, ich bin resolviert Sie einige pekene Reisen machen zu lassen. Sing Senjor, Sie nehmen unserm Herrn Ledermund, mais oh menus, die alten Pikaden ab, wir haben dort eine boa Fregesia, sing Senjor, Herr Ledermund soll nach Kaschwere, Strehle und andere Plätz gehen, und neue Turen arrangschieren, noh aeh. Ihre Reise ist kwasi nada, kwalker Kind könnte sie machen. Naturalmente muissen Sie, wenn Konkurrenten auf der Tur sind, semper na ponta sein, kleine Spesen, fix einkassieren, muito dinjero ist

29) Musterreiter, S. 44: Picapaos nannten die „Roten“ ihre Gegner nach den einläufigen Vogelflinten, mit denen deren freiwillige "Patriotas oft ausgerüstet waren. Auch der Spottname "Calombos" wurde für Regierungstruppen gebraucht; dies bezeichnet eine kurzhalsige Rasse Rindvieh, der jene - der Sage nach - beim Schlachten wegen besserm Fleischgeschmacks den Vorzug gaben. Die Bezeichnung "Maragatos" für die ,Roten“ bedeutet ,Spitzbuben“. 
bei mir das primaerschte, was ich sehn will, viele Fakturen, mas nur an ff Frigesen. Dass Sie mit grösster Promptigkeit reisen, aber naturalmente alle Kunden besuchen und wenigstens die Muster zeigen, ist certo. Beng klaro müssten Sie nach Ihrer Rückkehr Ihre naturalmente liegengebliebene Arbeit in den Büchern nachholen. Finalmente ist das poka poisa. Von Ihrem monatlichen Gehalt von $60 \$$ ziehe ich Ihnen nichts ab, entende, ich will Ihnen sogar noch $4 \$$ Reisespesen per Tag geben, uma koisa muito bong, noh aeh, so dass Sie sich in poko tempo eine schöne Oekonomie machen können. Das dient Ihnen, noh aeh? Sing Senhor, das wäre tudo; engdong até manjang. " ${ }^{30}$ ).

Portugiesische Spracheinflüsse im Werk des bekannten Heimatdichters Ernesto Niemeyer: „Die Ereignisse folgen sich schnell, und sie sind so wichtig, dass ich meinen Besuch bei Ihnen noch aufschieben muss.“ ${ }^{31}$ ). Da Niemeyer das Verb „folgen“ an einer anderen Stelle wiederum als reflexives gebraucht, kann es sich kaum um einen Druckfehler handeln: „Die Umarmung, die raschen, heissen Worte, die Küsse, alles folgte sich so schnell, dass er es wie in Betäubung geschehen liess." ${ }^{22}$ ). Hier hat die reflexive Verbform "seguir-se" bei Niemeyer Pate gestanden: Os acontecimentos seguem-se rapidamente. Die Redensart: "Ich sitze wie auf glühenden Kohlen" erscheint bei Niemeyer: „Ich stehe wie auf Kohlen." ${ }^{33}$ ). Vermutlich beruht sie auf dem portug. Sprachbild: "Eu estou como que sobre brasas".

Die zu diesem Thema gemachten Ausführungen erheben keineswegs das Prädikat der Lückenlosigkeit. Sie sollten lediglich die fliessenden Erscheinungsformen im brasilianischen Sprachraum festhalten. Aus dem Schrifttum zur deutschen Umgangssprache in Brasilien konnte nur ein Bruchteil herangezogen werden. Der aufgezeigte Anteil der Landessprache an der deutschen Tagessprache konnte nur die gebräulichsten

30) Musterreiter, S. 135 f.

31) Niemeyer, Ernesto: Solidor's Frauen-Ideal, ein brasilianischer Roman, S. 131.

32) Niemeyer, Solidor's Frauen-Ideal, S. 143.

33) rismeyer, Solidor's Frauen-Ideal, S. 106. 
Wörter erfassen, in der Meinung, dass sie überall in gleichem Grade Gültigkeit haben. Zu einer erschöpfenden Darstellung des Problems sind Einzelstudien lokalen und regionalen Charakters als Vorarbeiten für eine Gesamtschau, für ein Handbuch der deutschen Sprache in Brasilien, die Voraussetzung.

\section{Bibliographie zum Thema.}

Campos, Custódio F. de, Falares Blumenauenses, in: Centenário de Blumenau 1850-1950, Blumenau 1950, S. 373-386.

Die Arbeit enthält, neben einer Einführung, eine Aufstellung, in alphabetischer Anordnung, der Wörter zu diesem Thema mit genauen Quellenangaben im Schrifttum.

Löw, U., Neues Sprachgut und Sprachunsitten bei den Deutschen Rio Grandes, in: Serra-Post Kalender, Jhrg. 1927, Ijuhy - Santo Angelo, S. 97-107.

Der Aufsatz bietet viel sprachliches Material, meist regionalen Charakters, das später auch von Oberacker und Willems aufgenommen wurde.

Oberacker, C.H., Vocabulário de palavras portuguesas que os descendentes de colonos alemães acolheram na língua vulgar, in: Sociologia, Vol. 1, Nr. 3, São Paulo 1939, S. 96-104.

Schaden, Egon, Aculturação lingüística numa comunidade rural, in: Sociologia, Vol. IV. Nr. 3, São Paulo, 1942, S. 268-283. Neudruck davon in: Jornal de Filologia, Vol. I., Nr. 1, São Paulo, 1953; auch als Separatdruck, $18 \mathrm{~S}$.

Diese Schrift ist ein wertvoller wissenschaftlicher Beitrag zur Sprache in São Bonifácio, Santa Catarina. Das hier zusammengetragene Verzeichnis besteht aus 450 Termini.

Willems, Emilio, Assimiliação e populações marginais no Brasil, in: Bibliotéca Pedagógica Brasileira, Série 5, Vol. 186, Companhia Editora Nacional, S. Paulo, 1940, 343 S. Die Seiten 187-207 umfassen das Kapital über die 
Sprache, mit einer Aufstellung, in alphabetischer Reihenfolge, von 378 Wörtern.

Willems, Emilio, A aculturação dos alemães no Brasil, in: Bibliotéca Pedagógica Brasileira, Série 5, Vol. 250, Companhia Editora Nacional, São Paulo, 1946, 609 S. Zum Sprachproblem nimmt der Autor auf den S. 274-322 Stellung, in ausführlicherer Form als im oben zitierten Werk.

An Arbeiten, teilweise auch älteren Datums, die dem Verfasser nicht erreichbar waren, sind zu nennen:

Büchler, G.A., Verdeutschungsheft, Blumenau 1915.

Morais, Luiz Carlos de, Vocabulário Sul-Rio Grandense, Porto Alegre, 1935.

Wörterbuch für Deutsche Einwanderer, São Leopoldo, 1882. 